ESAIM: PROCEEDINGS AND SURVEYS, December 2017, Vol. 58, p. 78-97

Stéphane DELLACHERIE, Gloria FACCANONI, Bérénice GREC, Frédéric LAGOUTIERE, Yohan PENEL

\title{
MODELLING OF PHASE TRANSITIONS IN GRANULAR FLOWS
}

\author{
Charlotte Perrin ${ }^{1}$
}

\begin{abstract}
We present in this work a system for unidimensional granular flows first mentioned in a paper of A. LEFEBVRE-LEPot and B. MAURY (2011), which captures the transitions between compressible and incompressible phases. This model exhibits in the incompressible regions some memory effects through an additional variable called adhesion potential. We derive this system from compressible Navier-Stokes equations with singular viscosities and pressure, the singular limit between the two systems can then be seen as an analogue of the low Mach number limit for fluid with pressure dependent viscosity. It answers in a sense to the problem of transition between suspension flows and immersed granular flows identified by B. Andreotti, Y. Forterre and O. Pouliquen (2011). We illustrate the singular limit by numerical simulations.

Résumé. Dans cette étude nous présentons un modèle introduit à l'origine par A. LEFEBVRELEPOT et B. MAURY (2011) décrivant les transitions entre phases compressibles et incompressibles au sein d'écoulements granulaires unidimensionels. Ce modèle a la particularité d'introduire des effets de mémoire au sein des zones incompressible au travers d'une nouvelle variable appelée potentiel d'adhésion. Nous dérivons ce modèle mathématique à partir d'équations de Navier-Stokes compressibles où les lois de pression et de viscosités sont singulières au voisinage de la densité maximale. Le passage à la limite peut alors être vu comme un analogue d'une limite faible nombre de Mach locale pour des fluides avec viscosité dépendant de la pression. Ceci répond en un sens à la question posée par B. Andreotti, Y. Forterre et O Pouliquen (2011), d'une éventuelle transition entre écoulements de suspensions et écoulements granulaires. Nous illustrons ces résultats par des simulations numériques.
\end{abstract}

\section{INTRODUCTION}

We are interested in this paper in the macroscopic behaviour of a collection of solid particles immersed in a fluid. Our goal is to describe this system in the one-dimensional setting by means of continuous PDEs and precisely we want to model the transitions between the two states of the mixture: free when the density of solid particles is strictly less than 1; congested when the discrete particles are stuck to each other and the macroscopic density is equal to 1 . We are particularly interested in the derivation of such system from a regime of suspensions where the motion is dictated by the hydrodynamical interactions and lubrications forces. As explained by Andreotti, Forterre and Pouliquen in [1] it represents an important issue for the global understanding of granular media: "Note that the distinction between an immersed granular medium and a dense suspension dominated by hydrodynamic interactions is far from obvious and the question of a possible transition between a granular regime and a suspension regime remains open".

\footnotetext{
${ }^{1}$ LAMA UMR CNRS 5127, Université Savoie Mont-Blanc, 73376 Le Bourget-du-Lac, perrin@instmath.rwth-aachen.de
}

(c) EDP Sciences, SMAI 2017 
This question has been first addressed by Lefebvre-LePot and MaURY in [19], who derived from a microscopic system the following continuous one-dimensional model for suspensions

$$
\left\{\begin{array}{l}
\partial_{t} \rho+\partial_{x}(\rho u)=0 \\
\partial_{t}(\rho u)+\partial_{x}\left(\rho u^{2}\right)-\partial_{x}\left(\frac{\varepsilon}{1-\rho} \partial_{x} u\right)=\rho f
\end{array}\right.
$$

where the lubrication forces are taken into account through the singular viscous term. At the microscopic scale these forces prevent the contact between the solid particles. At the continuous scale, the singularity of the viscosity when the density gets closer to 1 , imposes the maximal constraint $\rho<1$. Letting then $\varepsilon$, the parameter representing the viscosity of the interstitial fluid, go to 0 , they propose an original limit two-phase system

$$
\left\{\begin{array}{l}
\partial_{t} \rho+\partial_{x}(\rho u)=0 \\
\partial_{t}(\rho u)+\partial_{x}\left(\rho u^{2}\right)+\partial_{x} p=\rho f \\
0 \leq \rho \leq 1 \\
\partial_{t} \gamma+\partial_{x}(\gamma u)=-p \\
(1-\rho) \gamma=0, \quad \gamma \leq 0
\end{array}\right.
$$

The pressure $p$ is then the limit of the lubrication forces $\frac{\varepsilon}{1-\rho} \partial_{x} u$ and is activated only in the congested regions. The idea of an additional variable $\gamma$ is inspired by the previous work of MAURY [20] in the case of a single particle which collides a solid wall. After the collision, the potential $\gamma$ is activated, takes negative values, and the particle remains glued until $\gamma$ hits 0 again. In the present situation, $\gamma$ reflects the same ideas of adhesion between the solid particles when there are stuck to each other, it keeps track of the constraints undergone by the particles in clusters.

We propose in this paper to justify rigorously the limit passage between systems for suspension flows with singular lubrication forces and systems for granular flows with transitions between compressible and incompressible phases and adhesion potential. For that purpose we introduce an additional viscosity which does not depend on the parameter $\varepsilon$ in order to apply classical energy and compactness methods. Compared to the mutidimensional case treated in [24], the analysis is simplified due to an additional entropy estimate usually called Mellet-Vasseur estimate. It gives thus a first mathematical answer to the physical intuition of ANDREOTTI, Forterre and Pouliquen [1] that "the rheology of immersed granular media and suspension could be described within the same framework".

We then illustrate the singular limit passage by designing a numerical scheme of the singular system for suspension flows. Our method relies on a previous work of DEGOND, HuA and NAVORET [11] concerning compressible Euler systems with singular pressure. The numerical simulations are in line with the theoretical results, we observe the good incompressible behaviour in the congested zones as $\varepsilon$ goes to 0 .

\section{Phase transitions In ONE-DIMENSIONAL GRANULAR FLOWS}

In [19], A. Lefebvre-Lepot and B. Maury introduce for the first time a system modelling transitions between compressible and incompressible phases inside granular flows. It reads as

$$
\left\{\begin{array}{l}
\partial_{t} \rho+\partial_{x}(\rho u)=0 \\
\partial_{t}(\rho u)+\partial_{x}\left(\rho u^{2}\right)+\partial_{x} p=\rho f \\
\partial_{t} \gamma+\partial_{x}(\gamma u)=-p \\
0 \leq \rho \leq 1 \\
(1-\rho) \gamma=0, \quad \gamma \leq 0
\end{array}\right.
$$


The free / compressible part, corresponding to the subdomain where $\{\rho<1\}$ is described by pressureless Euler equations, while in the congested part $\{\rho=1\}$ we have the incompressible constraint $\partial_{x} u=0$. Observe that in the congested domain, two additional variables are activated: the "pressure" $p$ and $\gamma$, called adhesion potential, which keeps track of the constraints undergone by the granular media.

A formal analysis of the system reveals the interesting structure provided by the adhesion potential and more precisely by its gradient. By deriving with respect to $x$ the third equation on $\gamma$ and using the fact $\gamma=0$ where $\rho<1$, we get

It leads us to consider the effective velocity

$$
\partial_{t}\left(\rho \partial_{x} \gamma\right)+\partial_{x}\left(\rho u\left(\partial_{x} \gamma\right)\right)=-\partial_{x} p
$$

$$
v_{\text {eff }}=u-\partial_{x} \gamma
$$

which coincides with $u$ outside the congested domain. Summing the momentum equation with the equation on the adhesion potential, the pressure contribution disappears from the new momentum equation

$$
\partial_{t}\left(\rho v_{\mathrm{eff}}\right)+\partial_{x}\left(\rho u v_{\mathrm{eff}}\right)=\rho f
$$

Testing then this equation by $v_{\text {eff }}$ and integrating in space we get

$$
\frac{\mathrm{d}}{\mathrm{d} t} \int_{\Omega} \frac{\rho\left|v_{\text {eff }}\right|^{2}}{2} \mathrm{~d} x \leq\left(\int_{\Omega} \rho|f|^{2} \mathrm{~d} x\right)^{2}\left(\int_{\Omega} \rho\left|v_{\text {eff }}\right|^{2} \mathrm{~d} x\right)
$$

and thus by using the bound on $\rho$ and a Gronwall inequality, we deduce the entropy inequality

$$
\sup _{t \in[0, T]} \int_{\Omega} \frac{\rho\left|v_{\text {eff }}\right|^{2}}{2} \mathrm{~d} x \leq C\left(\|f\|_{L^{2}}\right) \int_{\Omega} \frac{\rho^{0}\left|v_{\text {eff }}^{0}\right|^{2}}{2} \mathrm{~d} x .
$$

The system (1) can be compared with the pressureless Euler system under maximal constrained derived by Bouchut, Brenier, Cortes, Ripoll [3] and studied also by Berthelin in [2] and by Maury, Preux in [21] (where an additional non-elastic collision law is added)

$$
\left\{\begin{array}{l}
\partial_{t} \rho+\partial_{x}(\rho u)=0 \\
\partial_{t}(\rho u)+\partial_{x}\left(\rho u^{2}\right)+\partial_{x} \pi=0 \\
(1-\rho) \pi=0, \quad \pi \geq 0
\end{array}\right.
$$

where the memory effects in the congested domain are neglected and the adhesion potential is equal to 0.

Lefebvre-Lepot and Maury [19] present the biphasic system (1) as the formal limit of a compressible model where lubrication forces due to the viscosity of the interstitial fluid are taken into account. The system reads as

$$
\left\{\begin{array}{l}
\partial_{t} \rho_{\varepsilon}+\partial_{x}\left(\rho_{\varepsilon} u_{\varepsilon}\right)=0 \\
\partial_{t}\left(\rho_{\varepsilon} u_{\varepsilon}\right)+\partial_{x}\left(\rho_{\varepsilon} u_{\varepsilon}^{2}\right)-\partial_{x}\left(\lambda_{\varepsilon}\left(\rho_{\varepsilon}\right) \partial_{x} u_{\varepsilon}\right)=\rho_{\varepsilon} f
\end{array}\right.
$$

where the lubrication forces are known to be singular when the density of solid particles approaches 1: $\lambda_{\varepsilon}\left(\rho_{\varepsilon}\right)=$ $\frac{\varepsilon}{1-\rho_{\varepsilon}}$. It seems nevertheless not obvious to rigorously prove the compactness of the solutions $\left(\rho_{\varepsilon}, u_{\varepsilon}\right)$ to system (5) as $\varepsilon \rightarrow 0$, in particular in the convective term $\rho_{\varepsilon} u_{\varepsilon}^{2}$ and in the diffusion term $\frac{\varepsilon}{1-\rho_{\varepsilon}} \partial_{x} u_{\varepsilon}$. Indeed, the energy estimate provides

$$
\sup _{[0, T]} \int_{\Omega} \frac{\rho_{\varepsilon} u_{\varepsilon}^{2}}{2} \mathrm{~d} x+\varepsilon \int_{0}^{T} \int_{\Omega} \frac{1}{1-\rho_{\varepsilon}}\left(\partial_{x} u_{\varepsilon}\right)^{2} \mathrm{~d} x \mathrm{~d} t \leq C
$$


but does not yield any bound on the velocity $u_{\varepsilon}$ in the regions of low density where the viscosity tend to 0 . One can try to derive a similar structure as for the limit system by introducing the function $\varphi_{\varepsilon}$ such that $\varphi_{\varepsilon}^{\prime}\left(\rho_{\varepsilon}\right)=\frac{\lambda_{\varepsilon}\left(\rho_{\varepsilon}\right)}{\rho_{\varepsilon}}$ and which satisfies therefore

$$
\partial_{t}\left(\rho_{\varepsilon} \partial_{x} \varphi_{\varepsilon}\left(\rho_{\varepsilon}\right)\right)+\partial_{x}\left(\rho_{\varepsilon} \partial_{x} \varphi_{\varepsilon}\left(\rho_{\varepsilon}\right) u_{\varepsilon}\right)+\partial_{x}\left(\lambda_{\varepsilon}\left(\rho_{\varepsilon}\right) \partial_{x} u_{\varepsilon}\right)=0
$$

Then, it leads to the following equation on the effective velocity $v_{\varepsilon}=u_{\varepsilon}+\partial_{x} \varphi_{\varepsilon}\left(\rho_{\varepsilon}\right)$

$$
\partial_{t}\left(\rho_{\varepsilon} v_{\varepsilon}\right)+\partial_{x}\left(\rho_{\varepsilon} u_{\varepsilon} v_{\varepsilon}\right)=0
$$

where we recover the same structure as in (3). Unfortunately, the limit passage $\varepsilon \rightarrow 0$ using only these estimates seems out of reach due to the lack of control of $\partial_{x} \rho_{\varepsilon}$.

Remark: If, similarly to HAspot [15] we consider an initial velocity such that $u_{\varepsilon}^{0}=-\partial_{x} \varphi_{\varepsilon}\left(\rho_{\varepsilon}^{0}\right)$, i.e. $v_{\varepsilon}^{0}=0$, then the previous equation (via the energy inequality) gives that $u_{\varepsilon}=-\partial_{x} \varphi_{\varepsilon}\left(\rho_{\varepsilon}\right)$. By injecting then, the velocity $\left(\right.$ and $\varphi_{\varepsilon}$ ) into the mass equation, we recover a type of porous media equation

$$
\partial_{t} \rho_{\varepsilon}-\partial_{x}\left(\frac{\lambda_{\varepsilon}\left(\rho_{\varepsilon}\right)}{\rho_{\varepsilon}} \partial_{x} \rho_{\varepsilon}\right)=0
$$

which is also used in the modelling of thin films with singular pressure (see for instance BERTOZZI, GRÜN, Witelski [6]). Rewriting it as

$$
\partial_{t} \rho_{\varepsilon}-\partial_{x x}^{2} P_{\varepsilon}\left(\rho_{\varepsilon}\right)=0
$$

and expressing the evolution equation satisfied by $P_{\varepsilon}$

$$
\partial_{t} P_{\varepsilon}\left(\rho_{\varepsilon}\right)-P_{\varepsilon}^{\prime}\left(\rho_{\varepsilon}\right) \partial_{x x}^{2} P_{\varepsilon}\left(\rho_{\varepsilon}\right)=0
$$

we obtain a bound on $\partial_{x} P_{\varepsilon}$ in $L^{\infty}\left(0, T ; L^{2}(0,1)\right)$ (by testing the equation by $\left.-\partial_{x x}^{2} P_{\varepsilon}\right)$ if initially $\partial_{x} P_{\varepsilon}\left(\rho_{\varepsilon}^{0}\right)$ is bounded in $L^{2}$. In the spirit of the work of Perthame, Quiros, VAzQuez [25], it would be interesting to investigate a possible Hele-Shaw asymptotic as $\varepsilon \rightarrow 0$,

$$
\partial_{t} \rho-\partial_{x} Q=0, \quad \text { with } \quad(1-\rho) Q=0
$$

where $Q$ would be the weak limit of $\partial_{x} P_{\varepsilon}\left(\rho_{\varepsilon}\right)$. It is however not trivial to prove this limit passage and in particular the exclusion relation satisfied by $Q$. This work is in progress and will be the purpose of a forthcoming paper.

\section{Addition of extra viscosity}

We propose an alternative model for which we can rigorously prove the transition between a regime of suspension with lubrication forces and a regime of granular flows. It preserves the memory effects identified by LEFEBVRE-LEPot and MAURY and is also consistent with the theory of AndREOTTI, ForTERRE and Pouliquen [1] . As explained before the main obstacle in the previous system (1) is that it cannot be derived from the compressible system (5) via an energy method because we cannot rely our estimates on the singular viscosity appearing in (5). To avoid this difficulty, we add an additional viscosity of the form $\partial_{x}\left(\rho \partial_{x} u\right)$ which does not depend on $\varepsilon$. 
The model that we investigate throughout this paper is the following one

$$
\left\{\begin{array}{l}
\partial_{t} \rho+\partial_{x}(\rho u)=0 \\
\partial_{t}(\rho u)+\partial_{x}\left(\rho u^{2}\right)-\partial_{x} \gamma+\partial_{x} p-\mu_{0} \partial_{x}\left((\rho-\gamma) \partial_{x} u\right)=0 \\
0 \leq \rho \leq 1, \quad(1-\rho) \gamma=0, \quad \gamma \leq 0 \\
\partial_{t} \gamma+\partial_{x}(\gamma u)=-\frac{c_{\gamma}}{c_{\lambda}} p
\end{array}\right.
$$

defined on $\Omega=(0,1)$. For simplicity we will assume periodic boundary conditions.

In addition to the viscous term $-\partial_{x}\left(\rho \partial_{x} u\right)$ we have also added in (8) the pressure $-\partial_{x} \gamma$. Although this term is not crucial from a theoretical point of view, our numerical simulations in Section 3 strongly rely on this pressure. Remark that this additional pressure is activated only in the congested zones due to the exclusion relation $(1-\rho) \gamma=0$.

We introduce in the last equation two constants $c_{\gamma}, c_{\lambda}>0$ representing the dimension of $\gamma$ and $p$ respectively. We will see that effectively $p$ is the limit of the singular viscous forces $-\lambda_{\varepsilon}\left(\rho_{\varepsilon}\right) \partial_{x} u_{\varepsilon}$ and has not then the same physical interpretation as $\gamma$. The constants $c_{\gamma}, c_{\lambda}$ enable us to keep in mind the physical dimensions of the different quantities. Formally, letting $c_{\gamma} \rightarrow 0$ we recover with $\Pi=p$ the constraint system (4) (with additional viscosity). By reducing $c_{\gamma}$ we neglect thus $\gamma$ and the memory effects in the granular system.

The system (6) is the one-dimensional counterpart of the two-dimensional model introduced by the author in [24]. Remark that in dimension two, a turbulent drag term of the form $\alpha \rho|u| u$ was necessary to pass to the limit in the convective term $\rho u \otimes u$. In dimension one, as we will see below we can derive an additional estimate called the Mellet-Vasseur estimate which ensures the sufficient integrability of $\sqrt{\rho_{\varepsilon}}\left|u_{\varepsilon}\right|$ necessary to pass to limit and allows us to avoid such additional drag terms.

We propose to construct global weak solutions to (6) from a compressible system similar to (5) with singular lubrication forces.

Let the viscosity $\lambda_{\varepsilon}$ depend on the density of solid particles and such that

$$
\lim _{\rho \rightarrow 1}(1-\rho) \lambda_{\varepsilon}(\rho)>0
$$

In the domain $\Omega=(0,1)$ supplemented by periodic boundary conditions, we consider the singular system for suspension flows

$$
\left\{\begin{array}{l}
\partial_{t} \rho+\partial_{x}(\rho u)=0 \\
\partial_{t}(\rho u)+\partial_{x}\left(\rho u^{2}\right)-\partial_{x} \gamma_{\varepsilon}(\rho)-\partial_{x}\left(\left(\mu_{0} \rho-\frac{c_{\lambda}}{c_{\gamma}} \gamma_{\varepsilon}(\rho)\right) \partial_{x} u\right)-\partial_{x}\left(\lambda_{\varepsilon}(\rho) \partial_{x} u\right)=0 \\
0 \leq \rho<1
\end{array}\right.
$$

where the $\gamma_{\varepsilon}$ is related to $\lambda_{\varepsilon}$ through

$$
-\lambda_{\varepsilon}(\rho)=\frac{c_{\lambda}}{c_{\gamma}}\left(\gamma_{\varepsilon}^{\prime}(\rho) \rho-\gamma_{\varepsilon}(\rho)\right)
$$

Under condition (7), we ensure that $\gamma_{\varepsilon}(\rho)$ is also singular as $\rho$ approaches 1 . For technical reasons we assume additionally that for very small densities, $\gamma_{\varepsilon}(\rho)$ degenerates like $\rho$, i.e. that

$$
\gamma_{\varepsilon}(\rho) \underset{\rho \rightarrow 0}{\sim} C \rho
$$

Note that the total viscosity of the system has been thus split into two parts: the first one $\lambda_{\varepsilon}$ corresponds to the singular viscosity introduced by LEFEBVRE-LEPOT and MAURY; the additional one $\mu_{\varepsilon}(\rho)=\mu_{0} \rho-c_{\lambda} / c_{\gamma} \gamma_{\varepsilon}(\rho)$ 
is the one which will provide the entropy inequality that we need to pass to the limit $\varepsilon \rightarrow 0$.

Initially we assume that $\left(\rho_{\varepsilon}^{0}, m_{\varepsilon}^{0}\right)$ satisfies

$$
\begin{aligned}
& 0 \leq \rho_{\varepsilon}^{0}<1, \quad \partial_{x} \sqrt{\rho_{\varepsilon}^{0}}, \frac{\partial_{x} \gamma_{\varepsilon}\left(\rho_{\varepsilon}^{0}\right)}{\sqrt{\rho_{\varepsilon}^{0}}} \in L^{2}(0,1) \\
& \frac{\left|m_{\varepsilon}^{0}\right|^{2}}{\rho_{\varepsilon}^{0}}=0 \quad \text { a.e. on }\left\{x \in(0,1), \rho_{\varepsilon}^{0}=0\right\}, \quad \frac{\left|m_{\varepsilon}^{0}\right|^{2}}{\rho_{\varepsilon}^{0}} \in L^{1}(0,1)
\end{aligned}
$$

Theorem 1. Under the previous assumptions, there exists a subsequence of weak solutions $\left(\rho_{\varepsilon}, u_{\varepsilon}\right)$ to system $(8)$ such that

$$
\begin{aligned}
& \rho_{\varepsilon} \rightarrow \rho \text { in } \mathcal{C}([0, T] \times[0,1]) \\
& \sqrt{\rho_{\varepsilon}} u_{\varepsilon} \rightarrow \sqrt{\rho} u \quad \text { in } L^{2}((0, T) \times(0,1)) \\
& \sqrt{\rho_{\varepsilon}} \partial_{x} u_{\varepsilon} \rightarrow \sqrt{\rho} \partial_{x} u \quad \text { weakly in } L^{2}((0, T) \times(0,1)) \\
& \gamma_{\varepsilon}\left(\rho_{\varepsilon}\right) \rightarrow \gamma \quad \text { weakly-* } \text { in } L^{\infty}((0, T) \times(0,1)) \\
& \lambda_{\varepsilon}\left(\rho_{\varepsilon}\right) \partial_{x} u_{\varepsilon} \rightarrow-p \quad \text { weakly-* in } W^{-1, \infty}\left(0, T ; W^{-1,2}(0,1)\right)
\end{aligned}
$$

where $(\rho, u, \gamma, p)$ is a solution in the distributional sense to the granular system (6).

The relation (9) between $\gamma_{\varepsilon}$ and $\lambda_{\varepsilon}$ on the approximate system is crucial to pass to the limit in the equations. Indeed, it leads to the equation

$$
\partial_{t} \gamma_{\varepsilon}\left(\rho_{\varepsilon}\right)+\partial_{x}\left(\gamma_{\varepsilon}\left(\rho_{\varepsilon}\right) u_{\varepsilon}\right)=\frac{c_{\gamma}}{c_{\lambda}} \lambda_{\varepsilon}\left(\rho_{\varepsilon}\right) \partial_{x} u_{\varepsilon}
$$

which, as we will see later on, guarantees the compactness of the product $\lambda_{\varepsilon}\left(\rho_{\varepsilon}\right) \partial_{x} u_{\varepsilon}$. Note that this compactness could not be derived from the classical a priori estimates (energy, BD entropy) because of the singularity of the viscosity $\lambda_{\varepsilon}$ when $\rho_{\varepsilon}$ is close to 1 .

The singular limit passage between the compressible system (8) with singular pressure and viscosities and the final compressible/incompressible system (6) is then an analogue of a local low Mach number limit. The low Mach limit takes place in the regions where the approximate density $\rho_{\varepsilon}$ goes to 1 faster than $\varepsilon$ goes to 0 in such a way that the limit potential $\gamma=\lim \gamma_{\varepsilon}<0$. The critical rate of convergence being imposed by the order of the degeneracy of $\gamma_{\varepsilon}$ close to 1 . For instance, if

$$
\gamma_{\varepsilon}\left(\rho_{\varepsilon}\right)=-\varepsilon\left(\frac{\rho_{\varepsilon}}{1-\rho_{\varepsilon}}\right)^{\beta}, \quad \beta>0
$$

then the low Mach number limit takes place where $\rho_{\varepsilon}>1-\varepsilon^{1 / \beta}$.

\section{A priori estimates on the approximate system}

Let us briefly explain the a priori estimates necessary to obtain the convergence of weak solutions to system (8) towards weak solutions to (6). There are three inequalities to derive: the classical energy estimate, but also the so-called the BD and the Mellet-Vasseur entropy inequalities usually derived to prove the stability of weak solutions of compressible Navier-Stokes equations with density dependent viscosities. The first one will give us 
the strong convergence of the density $\rho_{\varepsilon}$ (see [7]) while the second gives a control of $\rho_{\varepsilon}\left|u_{\varepsilon}\right|^{2+\delta}$ necessary to pass to the limit in the product $\rho_{\varepsilon} u_{\varepsilon}^{2}$ (see [22]). For simplicity we will denote

$$
\begin{gathered}
\nu_{\varepsilon}(\rho)=\mu_{0} \rho+\lambda_{\varepsilon}(\rho)-\frac{c_{\lambda}}{c_{\gamma}} \gamma_{\varepsilon}(\rho) \\
\mu_{\varepsilon}(\rho)=\mu_{0} \rho-\frac{c_{\lambda}}{c_{\gamma}} \gamma_{\varepsilon}(\rho)
\end{gathered}
$$

- the energy inequality is given by

$$
\begin{aligned}
& \sup _{t \in[0, T]} \int_{0}^{1}\left[\frac{\rho_{\varepsilon}\left|u_{\varepsilon}\right|^{2}}{2}+\rho_{\varepsilon} e_{\varepsilon}\left(\rho_{\varepsilon}\right)\right]+\int_{0}^{T} \int_{0}^{1} \nu_{\varepsilon}\left(\rho_{\varepsilon}\right)\left|\partial_{x} u_{\varepsilon}\right|^{2} \\
& \leq \int_{0}^{1}\left[\frac{\rho_{\varepsilon}^{0}\left|u_{\varepsilon}^{0}\right|^{2}}{2}+\rho_{\varepsilon}^{0} e_{\varepsilon}\left(\rho_{\varepsilon}^{0}\right)\right]
\end{aligned}
$$

where $e_{\varepsilon}$ denotes the specific internal energy associated to $-\gamma_{\varepsilon}$ which is such that $e_{\varepsilon}^{\prime}(s)=-\gamma_{\varepsilon}(s) / s^{2}$. - BD entropy: thanks to the mass equation, equation (10) can be rewritten as

$$
\partial_{t}\left(\frac{c_{\lambda}}{c_{\gamma}} \gamma_{\varepsilon}\left(\rho_{\varepsilon}\right)-\mu_{0} \rho_{\varepsilon}\right)+\partial_{x}\left(\left(\frac{c_{\lambda}}{c_{\gamma}} \gamma_{\varepsilon}\left(\rho_{\varepsilon}\right)-\mu_{0} \rho_{\varepsilon}\right) u_{\varepsilon}\right)=\lambda_{\varepsilon}\left(\rho_{\varepsilon}\right) \partial_{x} u_{\varepsilon}
$$

or

$$
\partial_{t} \mu_{\varepsilon}\left(\rho_{\varepsilon}\right)+\partial_{x}\left(\mu_{\varepsilon}\left(\rho_{\varepsilon}\right) u_{\varepsilon}\right)=-\lambda_{\varepsilon}\left(\rho_{\varepsilon}\right) \partial_{x} u_{\varepsilon}
$$

Defining then $\Gamma_{\varepsilon}$ such that

$$
\Gamma_{\varepsilon}^{\prime}(\rho)=-\frac{\mu_{\varepsilon}^{\prime}(\rho)}{\rho}=\frac{\left(c_{\lambda} / c_{\gamma}\right) \gamma_{\varepsilon}^{\prime}(\rho)-\mu_{0}}{\rho}
$$

we get by derivation of the previous equation

$$
\partial_{t}\left(\rho_{\varepsilon} \partial_{x} \Gamma_{\varepsilon}\left(\rho_{\varepsilon}\right)\right)+\partial_{x}\left(\rho_{\varepsilon} \partial_{x} \Gamma_{\varepsilon}\left(\rho_{\varepsilon}\right) u_{\varepsilon}\right)-\partial_{x}\left(\mu_{\varepsilon}\left(\rho_{\varepsilon}\right) \partial_{x} u_{\varepsilon}\right)=\partial_{x}\left(\lambda_{\varepsilon}\left(\rho_{\varepsilon}\right) \partial_{x} u_{\varepsilon}\right)
$$

This leads us to consider the new velocity

$$
v_{\text {eff }}=u_{\varepsilon}-\partial_{x} \Gamma_{\varepsilon}\left(\rho_{\varepsilon}\right)
$$

which satisfies the simplified equation

$$
\partial_{t}\left(\rho_{\varepsilon} v_{\mathrm{eff}}\right)+\partial_{x}\left(\rho_{\varepsilon} u_{\varepsilon} v_{\mathrm{eff}}\right)-\partial_{x} \gamma_{\varepsilon}\left(\rho_{\varepsilon}\right)=0
$$

where the singular viscous terms have disappeared. Finally, testing the equation by $v_{\text {eff }}$ and combining it with (12) and the energy estimate we get

$$
\begin{aligned}
\sup _{t \in[0, T]} \int_{\Omega} & {\left[\frac{\rho_{\varepsilon}\left|v_{\text {eff }}\right|^{2}}{2}+\rho_{\varepsilon} e_{\varepsilon}\left(\rho_{\varepsilon}\right)\right] \mathrm{d} x+\int_{0}^{T} \int_{\Omega} \gamma_{\varepsilon}^{\prime}\left(\rho_{\varepsilon}\right) \Gamma_{\varepsilon}^{\prime}\left(\rho_{\varepsilon}\right)\left(\partial_{x} \rho_{\varepsilon}\right)^{2} \mathrm{~d} x \mathrm{~d} t } \\
\leq & \int_{\Omega}\left[\frac{\rho_{\varepsilon}^{0}\left|v_{\mathrm{eff}}^{0}\right|^{2}}{2}+\rho_{\varepsilon}^{0} e_{\varepsilon}\left(\rho_{\varepsilon}^{0}\right)\right] \mathrm{d} x
\end{aligned}
$$

As in the multi-dimensional work [24], we expect to control $\mu_{\varepsilon}$ thanks to this estimate, nevertheless the equation gives only a control of $\sqrt{\rho_{\varepsilon}} \partial_{x} \varphi_{\varepsilon}\left(\rho_{\varepsilon}\right)$. In order to use the compact embeddings between Sobolev 
spaces we have to guarantee a bound in $L^{1}$ of $\mu_{\varepsilon}$ which is not a consequence of the previous estimates. The idea is to combine the $\mathrm{BD}$ entropy with the estimate of $\mu_{\varepsilon}$

$$
\int_{0}^{1} \mu_{\varepsilon}\left(\rho_{\varepsilon}\right) \mathrm{d} x=\int_{0}^{t} \int_{0}^{1} \lambda_{\varepsilon}\left(\rho_{\varepsilon}\right) \partial_{x} u_{\varepsilon} \mathrm{d} x
$$

it remains then to control the right-hand side by integrating the momentum equation in time and twice in space. We do not reproduce the computations and refer to [24] for details. At the end, we get

$$
\begin{aligned}
& \sup _{t \in[0, T]} \int_{\Omega}\left[\frac{\rho_{\varepsilon}\left|v_{\mathrm{eff}}\right|^{2}}{2}+\rho_{\varepsilon} e_{\varepsilon}\left(\rho_{\varepsilon}\right)\right] \mathrm{d} x+\sup _{[0, T]} \int_{\Omega} \mu_{\varepsilon}\left(\rho_{\varepsilon}\right) \mathrm{d} x \\
& +\int_{0}^{T} \int_{\Omega} \gamma_{\varepsilon}^{\prime}\left(\rho_{\varepsilon}\right) \Gamma_{\varepsilon}^{\prime}\left(\rho_{\varepsilon}\right)\left(\partial_{x} \rho_{\varepsilon}\right)^{2} \mathrm{~d} x \mathrm{~d} t \leq C \int_{\Omega}\left[\frac{\rho_{\varepsilon}^{0}\left|v_{\mathrm{eff}}^{0}\right|^{2}}{2}+\rho_{\varepsilon}^{0} e_{\varepsilon}\left(\rho_{\varepsilon}^{0}\right)\right] \mathrm{d} x
\end{aligned}
$$

Remark (see details in [24]) that the computation also gives

$$
\lambda_{\varepsilon}\left(\rho_{\varepsilon}\right) \partial_{x} u_{\varepsilon} \text { bounded in } L^{1}((0, T) \times(0,1)) .
$$

- Mellet-Vasseur inequality: we test the momentum equation by $\left|u_{\varepsilon}\right|^{\delta} u_{\varepsilon}$ for $\delta>0$

$$
\int_{0}^{1}\left(\rho_{\varepsilon} \partial_{t} u_{\varepsilon}+\rho_{\varepsilon} u_{\varepsilon} \partial_{x} u_{\varepsilon}\right)\left|u_{\varepsilon}\right|^{\delta} u_{\varepsilon}-\int_{0}^{1} \partial_{x}\left(\nu_{\varepsilon}\left(\rho_{\varepsilon}\right) \partial_{x} u_{\varepsilon}\right)\left|u_{\varepsilon}\right|^{\delta} u_{\varepsilon}-\int_{0}^{1} \partial_{x} \gamma_{\varepsilon}\left(\rho_{\varepsilon}\right)\left|u_{\varepsilon}\right|^{\delta} u_{\varepsilon}=0
$$

Then

$$
\begin{aligned}
& \frac{1}{\delta+2} \int_{0}^{1} \rho_{\varepsilon} \partial_{t}\left(\left|u_{\varepsilon}\right|^{\delta+2}\right)+\frac{1}{\delta+2} \int_{0}^{1} \rho_{\varepsilon} u_{\varepsilon} \partial_{x}\left(\left|u_{\varepsilon}\right|^{\delta+2}\right)+(\delta+1) \int_{0}^{1} \nu_{\varepsilon}\left(\rho_{\varepsilon}\right)\left|u_{\varepsilon}\right|^{\delta}\left|\partial_{x} u_{\varepsilon}\right|^{2} \\
& \quad=-(\delta+1) \int_{0}^{1} \gamma_{\varepsilon}\left(\rho_{\varepsilon}\right)\left|u_{\varepsilon}\right|^{\delta} \partial_{x} u_{\varepsilon}
\end{aligned}
$$

using the mass equation and the energy estimate we get for $\eta \in(0,2)$

$$
\begin{aligned}
& \frac{1}{\delta+2} \frac{\mathrm{d}}{\mathrm{d} t} \int_{0}^{1} \rho_{\varepsilon}\left|u_{\varepsilon}\right|^{\delta+2}+(\delta+1) \int_{0}^{1} \nu_{\varepsilon}\left(\rho_{\varepsilon}\right)\left|u_{\varepsilon}\right|^{\delta}\left|\partial_{x} u_{\varepsilon}\right|^{2} \\
& \quad \leq C(\delta) \int_{0}^{1} \mu_{\varepsilon}\left(\rho_{\varepsilon}\right)\left(\partial_{x} u_{\varepsilon}\right)^{2}+C(\delta) \int_{0}^{1} \frac{\left|\gamma_{\varepsilon}\left(\rho_{\varepsilon}\right)\right|^{2}}{\mu_{\varepsilon}\left(\rho_{\varepsilon}\right)}\left|u_{\varepsilon}\right|^{2 \delta} \\
& \quad \leq C(\delta)+C(\delta)\left(\int_{0}^{1}\left(\frac{\left|\gamma_{\varepsilon}\left(\rho_{\varepsilon}\right)\right|^{2}}{\rho_{\varepsilon}^{\eta / 2} \mu_{\varepsilon}\left(\rho_{\varepsilon}\right)}\right)^{2 /(2-\eta)}\right)^{(2-\eta) / 2}\left(\int_{0}^{1} \rho_{\varepsilon}\left|u_{\varepsilon}\right|^{2 \delta / \eta}\right)^{\eta / 2} \\
& \quad \leq C(\delta)+C(\delta)\left(\int_{0}^{1}\left(\frac{\mu_{\varepsilon}\left(\rho_{\varepsilon}\right)}{\rho_{\varepsilon}^{\eta / 2}}\right)^{2 /(2-\eta) / 2}\left(\int_{0}^{1} \rho_{\varepsilon}\left|u_{\varepsilon}\right|^{2 \delta / \eta}\right)^{\eta / 2}\right.
\end{aligned}
$$

The integral of $\frac{\mu_{\varepsilon}\left(\rho_{\varepsilon}\right)}{\rho_{\varepsilon}^{\eta / 2}}$ is controlled thanks to the BD entropy (13). Indeed we control $\partial_{x} \mu_{\varepsilon}\left(\rho_{\varepsilon}\right)$ in $L^{\infty}\left(0, T ; L^{2}(0,1)\right), \mu_{\varepsilon}\left(\rho_{\varepsilon}\right)$ in $L^{1}((0, T) \times(0,1))$. By a Sobolev embedding $\mu_{\varepsilon}\left(\rho_{\varepsilon}\right)$ is then bounded in $L^{\infty}((0, T) \times(0,1))$. Since moreover $\mu_{\varepsilon}\left(\rho_{\varepsilon}\right)>\mu_{0} \rho_{\varepsilon}$ the ratio $\frac{\mu_{\varepsilon}\left(\rho_{\varepsilon}\right)}{\rho_{\varepsilon}^{\eta / 2}}$ is not singular close to the vacuum. 
The last integral is also controlled if we choose for instance $\delta \in(0,2)$ and $\eta=\delta$ since $\rho_{\varepsilon}\left|u_{\varepsilon}\right|^{2}$ is bounded in $L^{1}((0, T) \times(0,1))$ via the energy inequality $(11)$. If moreover we assume that initially

$$
\int_{0}^{1} \rho_{\varepsilon}^{0}\left|u_{\varepsilon}^{0}\right|^{\delta+2} \mathrm{~d} x \leq C
$$

we get therefore the Mellet-Vasseur inequality

$$
\sup _{t \in[0, T]} \int_{0}^{1} \rho_{\varepsilon}\left|u_{\varepsilon}\right|^{\delta+2} \mathrm{~d} x \leq C
$$

Remark: Compared to the multi-dimensional case treated in [24], the Mellet-Vasseur estimate is possible due to the positive sign of the integrals coming from the viscosities. In dimension two, testing the equation by $\left(1+\ln \left(1+\left|u_{\varepsilon}\right|^{2}\right)\right) u_{\varepsilon}$ we are not able to control the integral

$$
\begin{aligned}
& -\int_{\Omega} \nabla\left(\lambda_{\varepsilon}\left(\rho_{\varepsilon}\right) \operatorname{div} u_{\varepsilon}\right) \cdot\left(1+\ln \left(1+\left|u_{\varepsilon}\right|^{2}\right)\right) u_{\varepsilon} \\
& \quad=\int_{\Omega} \lambda_{\varepsilon}\left(\rho_{\varepsilon}\right)\left(1+\ln \left(1+\left|u_{\varepsilon}\right|^{2}\right)\right)\left(\operatorname{div} u_{\varepsilon}\right)^{2}+\int_{\Omega} \lambda_{\varepsilon}\left(\rho_{\varepsilon}\right) \frac{2 u_{\varepsilon}^{i} u_{\varepsilon}^{j}}{1+\left|u_{\varepsilon}\right|^{2}} \partial_{i} u_{\varepsilon}^{j} \operatorname{div} u_{\varepsilon}
\end{aligned}
$$

Indeed, the first integral has a positive sign but in the second, due to the cross derivatives $\partial_{i} u_{\varepsilon}^{k}$, we cannot use the control of $\lambda_{\varepsilon}\left(\rho_{\varepsilon}\right)\left(\operatorname{div} u_{\varepsilon}\right)^{2}$. Since $\lambda_{\varepsilon}\left(\rho_{\varepsilon}\right)$ is more singular than $\mu_{\varepsilon}\left(\rho_{\varepsilon}\right)$ as $\varepsilon \rightarrow 0$, we cannot control the integral by means of $\mu_{\varepsilon}\left(\rho_{\varepsilon}\right)$.

\section{Compactness arguments}

Thanks to (11), (13) and (15) we are then able to pass to the limit in the weak formulations of the equations. Except the convergence of the viscous term $\lambda_{\varepsilon}\left(\rho_{\varepsilon}\right) \partial_{x} u_{\varepsilon}$, the procedure is standard and follow the steps of [7] or [22] that we reproduce here for the convenience of the reader.

The control of $\sqrt{\rho_{\varepsilon}} v_{\text {eff }}=\sqrt{\rho_{\varepsilon}}\left(u_{\varepsilon}+\partial_{x} \mu_{\varepsilon}\left(\rho_{\varepsilon}\right)\right)$ given by the BD inequality (13) leads directly to the strong convergence of $\rho_{\varepsilon}$ and the weak convergence of $\gamma_{\varepsilon}\left(\rho_{\varepsilon}\right)$ towards $\rho$ and $\gamma$ respectively.

The next step of the proof consists in proving the strong convergence of $\rho_{\varepsilon} u_{\varepsilon}$ and $\sqrt{\rho_{\varepsilon}} u_{\varepsilon}$. First, we have from the energy estimate and the maximal constraint on $\rho_{\varepsilon}$ that $m_{\varepsilon}=\sqrt{\rho_{\varepsilon}} \sqrt{\rho_{\varepsilon}} u_{\varepsilon}$ is bounded in $L^{\infty}\left(0, T ; L^{2}(0,1)\right)$. By differentiation we have

from which we deduce that

$$
\partial_{x} m_{\varepsilon}=\underbrace{2 \partial_{x} \sqrt{\rho_{\varepsilon}}}_{\in L^{\infty} L^{2}} \underbrace{\sqrt{\rho_{\varepsilon}} u_{\varepsilon}}_{\in L^{\infty} L^{2}}+\underbrace{\sqrt{\rho_{\varepsilon}}}_{\in L^{\infty}} \underbrace{\sqrt{\rho_{\varepsilon}} \partial_{x} u_{\varepsilon}}_{\in L^{2} L^{2}}
$$

$$
\begin{aligned}
& \partial_{x} m_{\varepsilon} \quad \text { is bounded in } L^{2}\left(0, T ; L^{1}(0,1)\right), \\
& m_{\varepsilon} \quad \text { is bounded in } L^{2}\left(0, T ; W^{1,1}(0,1)\right) .
\end{aligned}
$$

On the other hand, thanks to the bound on $\lambda_{\varepsilon}\left(\rho_{\varepsilon}\right) \partial_{x} u_{\varepsilon}$ in $L^{1}((0, T) \times(0,1))$, the analysis of the momentum equation gives that

$$
\partial_{t} m_{\varepsilon} \quad \text { is bounded in } L^{1}\left(0, T ; W^{-1,1}(0,1)\right) .
$$

We can then apply the Aubin-Lions-Simon Lemma (see [5]) to the sets $B_{0}=W^{1,1}(0,1), B_{1}=L^{p}(0,1)(p \in$ $[1,+\infty))$ and $B_{2}=W^{-1,1}(0,1)$ : we have the continuous embeddings of $B_{0}$ in $B_{1}$ and of $B_{1}$ in $B_{2}$ and the compact embedding of $B_{0}$ in $B_{1}$. Finally we deduce that there exists $m$ such that

$$
m_{\varepsilon} \longrightarrow m \quad \text { a.e. in } \quad(0, T) \times(0,1)
$$


and strongly in $L^{2}\left(0, T ; L^{p}(0,1)\right)$ for all $p \in[1,+\infty)$.

Now, since $m_{\varepsilon} / \sqrt{\rho_{\varepsilon}}=\sqrt{\rho_{\varepsilon}} u_{\varepsilon}$ is bounded $L^{\infty}\left(0, T ; L^{2}(0,1)\right)$, by the Fatou lemma

$$
\int_{\Omega} \liminf \frac{m_{\varepsilon}^{2}}{\rho_{\varepsilon}} \mathrm{d} x \leq \liminf \int_{\Omega} \frac{m_{\varepsilon}^{2}}{\rho_{\varepsilon}} \mathrm{d} x \leq C
$$

and we deduce that

$$
m(t, x)=0 \quad \text { on } \quad\{\rho(t, x)=0\} .
$$

We can thus define a limit velocity $u$ by

$$
u(t, x)=\left\{\begin{array}{lll}
\frac{m(t, x)}{\rho(t, x)} & \text { if } & \rho(t, x) \neq 0 \\
0 & \text { if } & \rho(t, x)=0
\end{array}\right.
$$

The Mellet-Vasseur estimate (15) provides a bound of $\rho_{\varepsilon}\left|u_{\varepsilon}\right|^{\delta+2}$ in $L^{\infty}\left(0, T ; L^{1}(0,1)\right)$ necessary to prove the strong convergence of $\sqrt{\rho_{\varepsilon}} u_{\varepsilon}$ in $L^{2}((0, T) \times(0,1))$. Let us explain more precisely this point. Thanks to the Fatou Lemma we ensure that the limit $\rho|u|^{\delta+2}$ is bounded in $L^{\infty}\left(0, T ; L^{1}(0,1)\right)$. To prove the strong convergence of $\sqrt{\rho_{\varepsilon}} u_{\varepsilon}$ we introduce a constant $M>0$ and consider separately the small velocities $|u| \leq M$ and the large velocities $|u|>M$

$$
\begin{aligned}
& \int_{0}^{T} \int_{0}^{1}\left|\sqrt{\rho_{\varepsilon}} u_{\varepsilon}-\sqrt{\rho} u\right|^{2} \\
& \quad \leq 2 \int_{0}^{T} \int_{0}^{1}\left|\sqrt{\rho_{\varepsilon}} u_{\varepsilon} \mathbf{1}_{\left\{\left|u_{\varepsilon}\right| \leq M\right\}}-\sqrt{\rho} u \mathbf{1}_{\{|u| \leq M\}}\right|^{2} \\
& \quad+4 \int_{0}^{T} \int_{0}^{1} \rho_{\varepsilon}\left|u_{\varepsilon}\right|^{2} \mathbf{1}_{\left\{\left|u_{\varepsilon}\right|>M\right\}}+4 \int_{0}^{T} \int_{0}^{1} \rho|u|^{2} \mathbf{1}_{\{|u|>M\}}
\end{aligned}
$$

Thanks to the a.e. convergence of $\rho_{\varepsilon}, m_{\varepsilon}$ and the definition of $u$ at the limit, we have

$$
\sqrt{\rho_{\varepsilon}} u_{\varepsilon} \mathbf{1}_{\left\{\left|u_{\varepsilon}\right| \leq M\right\}} \longrightarrow \sqrt{\rho} u \mathbf{1}_{\{|u| \leq M\}} \quad \text { a.e. on } \quad\{(t, x), \rho(t, x) \neq 0\}
$$

and on the other hand

$$
\sqrt{\rho_{\varepsilon}} u_{\varepsilon} \mathbf{1}_{\left\{\left|u_{\varepsilon}\right| \leq M\right\}} \leq M \sqrt{\rho_{\varepsilon}} \rightarrow 0 \quad \text { a.e. on } \quad\{(t, x), \rho(t, x)=0\}
$$

due to the convergence a.e. of $\rho_{\varepsilon}$. Then, the dominated convergence theorem gives the convergence of the first integral of (17) towards 0. For the two remaining integrals we use the bound given by the Mellet-Vasseur estimate to obtain

$$
\begin{aligned}
\int_{0}^{T} \int_{0}^{1} \rho_{\varepsilon}\left|u_{\varepsilon}\right|^{2} \mathbf{1}_{\left\{\left|u_{\varepsilon}\right|>M\right\}} & \leq \int_{0}^{T} \int_{0}^{1} \rho_{\varepsilon} \frac{\left|u_{\varepsilon}\right|^{\delta+2}}{\left|u_{\varepsilon}\right|^{\delta}} \mathbf{1}_{\left\{\left|u_{\varepsilon}\right|>M\right\}} \\
& \leq \frac{1}{M^{\delta}} \int_{0}^{T} \int_{0}^{1} \rho_{\varepsilon}\left|u_{\varepsilon}\right|^{\delta+2} \mathbf{1}_{\left\{\left|u_{\varepsilon}\right|>M\right\}} \\
& \underset{M}{\longrightarrow} 0
\end{aligned}
$$

and, coming back to (17),

$$
\sqrt{\rho_{\varepsilon}} u_{\varepsilon} \longrightarrow \sqrt{\rho} u \quad \text { strongly in } L^{2}((0, T) \times(0,1))
$$


With the previous convergence we can then pass to the limit into the diffusion term $\partial_{x}\left(\mu_{\varepsilon}\left(\rho_{\varepsilon}\right) \partial_{x} u_{\varepsilon}\right)$. The weak formulation writes as

$$
\begin{aligned}
\int_{0}^{T} \int_{0}^{1} \partial_{x}\left(\mu_{\varepsilon}\left(\rho_{\varepsilon}\right) \partial_{x} u_{\varepsilon}\right) \xi & =-\int_{0}^{T} \int_{0}^{1} \mu_{\varepsilon}\left(\rho_{\varepsilon}\right) \partial_{x} u_{\varepsilon} \partial_{x} \xi \\
& =\int_{0}^{T} \int_{0}^{1} \frac{\partial_{x} \mu_{\varepsilon}\left(\rho_{\varepsilon}\right)}{\sqrt{\rho_{\varepsilon}}} \sqrt{\rho_{\varepsilon}} u_{\varepsilon} \partial_{x} \xi+\int_{0}^{T} \int_{0}^{1} \frac{\mu_{\varepsilon}\left(\rho_{\varepsilon}\right)}{\sqrt{\rho_{\varepsilon}}} \sqrt{\rho_{\varepsilon}} u_{\varepsilon} \partial_{x x}^{2} \xi
\end{aligned}
$$

for $\xi \in \mathcal{D}((0, T) \times(0,1))$. We can then check that $\frac{\mu_{\varepsilon}\left(\rho_{\varepsilon}\right)}{\sqrt{\rho_{\varepsilon}}}$ and $\frac{\partial_{x} \mu_{\varepsilon}\left(\rho_{\varepsilon}\right)}{\sqrt{\rho_{\varepsilon}}}$ converge weakly in $L^{2}((0, T) \times(0,1))$ towards $\frac{\mu_{0} \rho-c_{\lambda} / c_{\gamma} \gamma}{\sqrt{\rho}}$ and $\frac{\partial_{x}\left(\mu_{0} \rho-c_{\lambda} / c_{\gamma} \gamma\right)}{\sqrt{\rho}}$ respectively.

We finally pass to the limit in the singular viscous term $\partial_{x}\left(\lambda_{\varepsilon}\left(\rho_{\varepsilon}\right) u_{\varepsilon}\right)$. Contrary to $\mu_{\varepsilon}$, the coefficient $\lambda_{\varepsilon}\left(\rho_{\varepsilon}\right)$, alone, is not controlled, but the product $\lambda_{\varepsilon}\left(\rho_{\varepsilon}\right) \partial_{x} u_{\varepsilon}$ is. To conclude we come back to the additional equation

$$
\partial_{t} \gamma_{\varepsilon}\left(\rho_{\varepsilon}\right)+\partial_{x}\left(\gamma_{\varepsilon}\left(\rho_{\varepsilon}\right) u_{\varepsilon}\right)=\frac{c_{\gamma}}{c_{\lambda}} \lambda_{\varepsilon}\left(\rho_{\varepsilon}\right) \partial_{x} u_{\varepsilon}
$$

from which we deduce that

$$
p_{\varepsilon} \quad \text { is bounded in } \quad W^{-1, \infty}\left(0, T ; L^{\infty}(0,1)\right)+L^{\infty}\left(0, T ; W^{-1,2}(0,1)\right) .
$$

There exists thus $p$ such that

$$
p_{\varepsilon}=\lambda_{\varepsilon}\left(\rho_{\varepsilon}\right) \partial_{x} u_{\varepsilon} \longrightarrow p \quad \text { weakly-* in } \quad W^{-1, \infty}\left(0, T ; L^{\infty}(0,1)\right)+L^{\infty}\left(0, T ; W^{-1,2}(0,1)\right)
$$

We can then pass to the weak limit both in (8) and in the additional equation (10) to get the result of the theorem.

Remark: The important issue would be now to pass to the limit $\mu_{0} \rightarrow 0$ in the final equations (6) to recover the system (1) introduced by Lefebvre-Lepot and MaUry. This limit passage seems not possible by means of the previous compactness arguments because the additional viscosity provides the necessary bounds for the strong convergence of the density.

Another possibility but which is beyond the scope of this paper, would be the adaptation of compactness arguments for the vanishing viscosity limit in pressureless systems used by Boudin in [4]. This work is in progress and will be the purpose of a forthcoming paper.

\section{Numerical Simulation}

We propose in this section to simulate the singular compressible system (8) for small values of $\varepsilon$. Our goal is to find a good discretization able to capture the behaviour of the solutions of the biphasic system (6) as $\varepsilon \rightarrow 0$. Bringing together all the viscous terms, the system rewrites as

$$
\left\{\begin{array}{l}
\partial_{t} \rho_{\varepsilon}+\partial_{x}\left(\rho_{\varepsilon} u_{\varepsilon}\right)=0 \\
\partial_{t}\left(\rho_{\varepsilon} u_{\varepsilon}\right)+\partial_{x}\left(\rho_{\varepsilon} u_{\varepsilon}^{2}\right)-\partial_{x} \gamma_{\varepsilon}\left(\rho_{\varepsilon}\right)-\partial_{x}\left(\nu_{\varepsilon}\left(\rho_{\varepsilon}\right) \partial_{x} u_{\varepsilon}\right)=0
\end{array}\right.
$$

where

$$
\nu_{\varepsilon}\left(\rho_{\varepsilon}\right)=\mu_{0} \rho_{\varepsilon}-\frac{c_{\lambda}}{c_{\gamma}} \gamma_{\varepsilon}^{\prime}\left(\rho_{\varepsilon}\right) \rho_{\varepsilon}
$$


To simplify we will consider a potential $\gamma_{\varepsilon}$ such that

$$
\gamma_{\varepsilon}(\rho)=-\varepsilon\left(\frac{\rho}{1-\rho}\right)^{\beta}, \quad \beta>1
$$

\section{Congestion}

The first property that our numerical scheme has to guarantee, is the maximal density constraint $\rho_{\varepsilon}<1$. We will introduce in our scheme a specific splitting and time discretization of the singular pressure which ensures that this constraint is automatically satisfied.

Even though the maximal constraint cannot be reached by the numerical solution, we have an approximation of the phase transitions in the limit system. Indeed if the approximate density is such that $1-\rho_{\varepsilon}<\varepsilon^{1 / \beta}$ then the limit potential $\gamma=\lim _{\varepsilon} \gamma_{\varepsilon}$ is activated. Restricting thus the notion of a congested region on the limit system as a region where $\gamma<0$ we can define at $\varepsilon$ fixed quasi congested regions as the regions where $\left\{\rho_{\varepsilon}>1-\varepsilon^{1 / \beta}\right\}$. If $\varepsilon$ is small enough and if our scheme is not too much diffusive when the density is high, we can expect from our previous theoretical results to observe approximated phase transitions on the numerical solutions.

\section{Difficulties}

We are looking for a numerical scheme able to capture the limit behaviour given by equations (6) as $\varepsilon \rightarrow 0$ and especially the phase transitions between congested and uncongested zones. This problem mixes then several scales, the reference macroscopic scale and the small scale $\varepsilon^{1 / \beta}$ which encodes the transitions between the compressible and the incompressible zones. Generally speaking we ask for the stability of the scheme as $\varepsilon \rightarrow 0$, meaning the discretization should not depend on $\varepsilon$, and for the asymptotic consistency with equations (6). This is the so-called Asymptotic Preserving (AP) property introduced by JIN [17].

Our problem is closely related to dealing with low Mach numbers in compressible systems. Let us mention the global ideas of the most commonly used techniques on this problem. It is a well-known fact that techniques employed for conservative compressible systems fail to reproduce the incompressible behaviour in domains where the Mach number goes to 0 and the speed of the acoustic waves becomes very large. Several ideas have been proposed to tackle this issue among which preconditioning methods (see [28]) which consist in multiplying the time derivatives by an appropriate matrix in order to alter the stiff eigenvalues of the system, and pressure correction methods, which extend to the compressible setting the projection techniques introduced by CHORIN [8], TEMAM [27] in the incompressible framework (see for instance the works of HARLOW, AMSDEN [14] or more recently the works of HeRBIn, KhERIJI, LATChÉ [16]). We are interested in this last category and more precisely in the recent techniques of implicit/explicit (IMEX) discretizations proposed for instance by KLEIN [18], Degond et al. [10], Degond and Tang [13], Cordier, Degond, Kumbaro [9], Noelle et al. [23]. These methods consist in splitting the pressure into a stiff and a non-stiff part, the first one being treated implicitly in time whereas the non-stiff part is treated explicitly. The implicit treatment of the stiff part enables then the stability with respect to the propagation of the acoustic waves.

\section{IMEX splitting of the pressure}

Usually the splitting of the pressure relies on physical arguments as explained by Klein [18] and depends specifically of the considered situation. In our case we need to capture the "almost" incompressible behaviour of the solution in congested regions but also the "almost" pressureless dynamics in uncongested regions. This 
specific issue has been adressed by Degond, HuA and NAvoret in [11] for the singular Euler system

$$
\left\{\begin{array}{l}
\partial_{t} \rho+\operatorname{div}(\rho u)=0 \\
\partial_{t}(\rho u)+\operatorname{div}(\rho u \otimes u)+\varepsilon \nabla\left(\frac{1}{\frac{1}{\rho}-\frac{1}{\rho^{*}}}\right)^{\gamma}=0 \\
0 \leq \rho \leq \rho^{*}
\end{array}\right.
$$

We propose here to base our numerical investigations of the viscous system (19) on the IMEX scheme introduced by Degond, Hua and Navoret by splitting our scheme into two steps: first we solve of the Euler part of the system, then we add the effect of the viscosity by solving a heat equation. Let us briefly explain the idea developed in [11] that we reproduce for the inviscid part of our system

$$
\left\{\begin{array}{l}
\partial_{t} \rho_{\varepsilon}+\partial_{x}\left(\rho_{\varepsilon} u_{\varepsilon}\right)=0 \\
\partial_{t}\left(\rho_{\varepsilon} u_{\varepsilon}\right)+\partial_{x}\left(\rho_{\varepsilon} u_{\varepsilon}^{2}\right)-\partial_{x} \gamma_{\varepsilon}\left(\rho_{\varepsilon}\right)=0
\end{array}\right.
$$

which is hyperbolic. Its characteristic speeds

$$
u_{\varepsilon} \pm \sqrt{-\left(\gamma_{\varepsilon}^{\prime}\left(\rho_{\varepsilon}\right)\right)}=u_{\varepsilon} \pm \sqrt{\varepsilon \frac{\rho_{\varepsilon}^{\beta-1}}{\left(1-\rho_{\varepsilon}\right)^{\beta+1}}}
$$

are not bounded uniformly with respect to $\varepsilon$ since we may have in the approximated congested zones $1-\rho_{\varepsilon}<$ $\varepsilon^{s}<\varepsilon^{1 /(\beta+1)}$. This implies for classical explicit schemes a stringent Courant-Friedrichs-Levy (CFL) condition

$$
\Delta t \leq \frac{\sigma \Delta x}{\max \left|u_{\varepsilon}\right|+\sqrt{\left|\gamma_{\varepsilon}^{\prime}\left(\rho_{\varepsilon}\right)\right|}}, \quad 0 \leq \sigma \leq 1
$$

and imposes very small time steps $\Delta t$. A fully explicit scheme will be then inefficient even for reasonably small values of $\varepsilon$. It is known also that a full implicit discretization of the pressure may be also problematic because it induces a lot of diffusion. As previously said, the idea is then to mix an explicit and an implicit treatment of the pressure. We decompose thus the potential $\gamma_{\varepsilon}$ into two parts

$$
\gamma_{\varepsilon}(\rho)=\gamma_{\varepsilon}^{\mathrm{im}}(\rho)+\gamma_{\varepsilon}^{\mathrm{ex}}(\rho)
$$

which depend on an additional parameter denoted $\delta$

$$
\gamma_{\varepsilon}^{\mathrm{ex}}(\rho)= \begin{cases}\frac{\gamma_{\varepsilon}(\rho)}{2} & \text { if } \quad \rho \leq 1-\delta \\ \frac{1}{2}\left[\gamma_{\varepsilon}(1-\delta)+(\rho-1+\delta) \gamma_{\varepsilon}^{\prime}(1-\delta)+\frac{1}{2}(\rho-1+\delta)^{2} \gamma_{\varepsilon}^{\prime \prime}(1-\delta)\right] & \text { if } \quad \rho>1-\delta\end{cases}
$$

In order to ensure that the contribution of $\gamma_{\varepsilon}^{\text {ex }}$ in the elliptic equation satisfied by the pressure (see Equation (22)) remains bounded with respect to $\varepsilon$, we need to choose $\delta$ big enough such that the second derivative of $\gamma_{\varepsilon}^{\text {ex }}$ remains bounded with respect to $\varepsilon$. An appropriate choice is then

$$
\delta=\varepsilon^{1 /(\beta+2)} .
$$

The second part $\gamma_{\varepsilon}^{\mathrm{im}}$, defined as the difference between the total potential $\gamma_{\varepsilon}$ and $\gamma_{\varepsilon}^{\text {ex }}$, supports the singularity close to $\rho=1$. We illustrate this splitting on Figure 1. The idea is now to treat implicitly in time the singular 


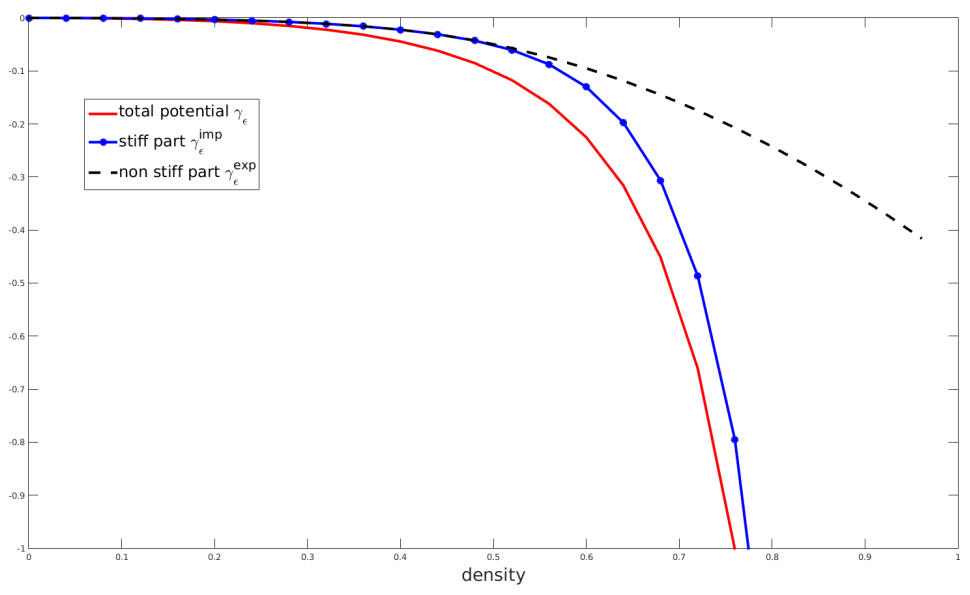

FiguRe 1. Splitting of $\gamma_{\varepsilon}$ into a singular part $\gamma_{\varepsilon}^{\mathrm{im}}$ and a bounded one $\gamma_{\varepsilon}^{\mathrm{ex}}, \beta=2, \varepsilon=0.1, \delta=\varepsilon^{1 /(\beta+2)}$

part $\gamma_{\varepsilon}^{\text {im }}$ and explicitly $\gamma_{\varepsilon}^{\text {ex }}$. The CFL condition computed from $\gamma_{\varepsilon}^{\text {ex }}$

$$
\frac{\Delta t}{\Delta x} \leq \frac{\sigma}{\max \left|u_{\varepsilon}\right|+\sqrt{\left|\left(\gamma_{\varepsilon}^{\mathrm{ex}}\right)^{\prime}\left(\rho_{\varepsilon}\right)\right|}}, \quad 0 \leq \sigma \leq 1
$$

is thus no longer stiff which avoids the time step restrictions that we had on the original system.

\section{Discretization of the equations}

We introduce $\rho^{n}, u^{n}$ and $v^{n+1 / 2}$ the numerical approximations of the density $\rho$, the velocity of the full system $u$ and $v$ the velocity of the hyperbolic part of the system respectively, at the discrete times $t^{n}=n \Delta t, t^{n+1 / 2}=$ $(n+1 / 2) \Delta t, \Delta t$ being the time step. We describe in the sequel only the time-discretization of the equation and refer to [11] for the full discretization in time and space. First we solve $\left(\rho^{n+1 / 2}, q^{n+1 / 2}=\rho^{n+1 / 2} v^{n+1 / 2}\right)$ the numerical solution of the Euler part, by treating implicitly the flux in the mass equation and only the singular pressure $\gamma_{\varepsilon}^{\text {im }}$ in the momentum equation

$$
\left\{\begin{array}{l}
\frac{\rho^{n+1 / 2}-\rho^{n}}{\Delta t / 2}+\partial_{x}\left(q^{n+1 / 2}\right)=0 \\
\frac{q^{n+1 / 2}-q^{n}}{\Delta t / 2}+\partial_{x}\left(\frac{\left(q^{n}\right)^{2}}{\rho^{n}}\right)-\partial_{x}\left(\gamma_{\varepsilon}^{\operatorname{ex}}\left(\rho^{n}\right)\right)-\partial_{x}\left(\gamma_{\varepsilon}^{\operatorname{im}}\left(\rho^{n+1 / 2}\right)\right)=0
\end{array}\right.
$$

and then we will have to compute the velocity $u^{n+1}$ from the density $\rho^{n+1}=\rho^{n+1 / 2}$ and $q^{n+1 / 2}=(\rho v)^{n+1 / 2}$, by taking into account the viscosity.

Concerning the solving of the hyperbolic part, the first step consists in a reformulation of the system to obtain the elliptic equation satisfied by the singular pressure $\left(\gamma_{\varepsilon}^{\mathrm{im}}\right)^{n+1 / 2}$. For that purpose we replace the momentum $q^{n+1 / 2}$ in (21a) by taking the divergence of the momentum equation (21b). Seeing the density as a function of 
this potential $\rho^{n+1 / 2}=\rho\left(\left(\gamma_{\varepsilon}^{\mathrm{im}}\right)^{n+1 / 2}\right)$, we obtain a nonlinear elliptic equation on $\gamma_{\varepsilon}^{\mathrm{im}}$

$$
\begin{aligned}
& \rho\left(\left(\gamma_{\varepsilon}^{\mathrm{im}}\right)^{n+1 / 2}\right)+\left(\frac{\Delta t}{2}\right)^{2} \partial_{x x}^{2}\left(\gamma_{\varepsilon}^{\mathrm{im}}\right)^{n+1 / 2} \\
& \quad=\rho^{n}-\frac{\Delta t}{2} q^{n}+\left(\frac{\Delta t}{2}\right)\left[\partial_{x x}^{2}\left(\left(q^{n}\right)^{2} / \rho^{n}\right)-\partial_{x x}^{2} \gamma_{\varepsilon}^{\mathrm{ex}}\left(\rho^{n}\right)\right]
\end{aligned}
$$

The solving of this non-linear equation is performed by means of a quasi-Newton method.

Once this pressure $\left(\gamma_{\varepsilon}^{\mathrm{im}}\right)^{n+1 / 2}$ is computed, we invert numerically (as we did in the previous Newton iterations) the function $\rho \mapsto \gamma_{\varepsilon}^{\operatorname{im}}(\rho)$, which gives us the new density $\rho^{n+1 / 2}$ which satisfies automatically the maximal constraint $\rho^{n+1 / 2}<1$. This is also done via the Newton method.

To compute the momentum $q^{n+1 / 2}$ of this Euler system, two methods are possible. The first one consists in solving directly the momentum equation, while the second relies on the Gauge decomposition of the momentum

$$
q=a-\partial_{x} \phi
$$

where $a$ is the divergence-free part, i.e. constant in our one-dimensional setting, and $\phi$ is the irrotational part. The numerical test cases done in [11] show that both methods have drawbacks. The direct method creates oscillations on the velocity in congested part, while the Gauge method is diffusive in uncongested part. Since the last method is expected to be more efficient to capture the transitions between the congested and the uncongested regions, we propose to implement it for our simulations.

We compute numerically

$$
\begin{aligned}
& \partial_{x x}^{2} \phi^{n+1 / 2}=\frac{1}{\Delta t / 2}\left(\rho^{n+1 / 2}-\rho^{n}\right) \\
& a^{n+1 / 2}=a^{n}-\frac{\Delta t}{2}\left[\frac{\left(q^{n}\right)^{2}}{\rho^{n}}-\gamma_{\varepsilon}^{\operatorname{ex}}\left(\rho^{n}\right)-\gamma_{\varepsilon}^{\operatorname{im}}\left(\rho^{n+1 / 2}\right)\right]_{x=0}^{x=1}
\end{aligned}
$$

which gives the new momentum

$$
\rho^{n+1 / 2} v^{n+1 / 2}=q^{n+1 / 2}=a^{n+1 / 2}-\partial_{x} \phi^{n+1 / 2}
$$

We have imposed additionally periodic boundary conditions to solve (22) and (23).

We do not write the full discretization in space for the Euler system (21) which is exactly the one used by DEGOND, HuA and NAvoret, namely a local Lax-Friedrichs scheme with a centered flux to which we also add numerical diffusion. We refer to [11] concerning the choice of the artificial numerical diffusion introduced in the numerical fluxes. In particular this choice imposes additionally that

$$
\frac{\Delta t}{\Delta x} \geq \frac{1}{2\left(\sqrt{\left|\left(\gamma_{\varepsilon}^{\mathrm{ex}}\right)^{\prime}\left(\rho^{n}\right)\right|}+\sqrt{\left|\gamma_{\varepsilon}^{\prime}\left(\rho^{n}\right)\right|}\right)}
$$

in order to damp out the numerical oscillations.

Concerning the viscosity that we did not treat yet, we compute now the new velocity $u^{n+1}$ from $\rho^{n+1}=\rho^{n+1 / 2}$ and $v^{n+1 / 2}$ with the equation

$$
\rho^{n+1} \frac{u^{n+1}-v^{n+1 / 2}}{\Delta t / 2}-\partial_{x}\left(\nu\left(\rho^{n+1}\right) \partial_{x} u^{n+1}\right)=0
$$

We have treated implicitly the diffusion term, because the viscosity $\mu$ tends to $+\infty$ close to the congestion constraint and this would lead to a too much restrictive stability condition if it is treated explicitly. 


\section{Discussion of the asymptotic consistency}

The splitting that we employed which consists in treating separately the viscosity from the hyperbolic part is expected to be diffusive in the quasi-congested regions where the singular viscosity is activated. The question is to determine whether this diffusion impacts the asymptotic consistency of the scheme. Obviously the numerical scheme is adjusted for the hyperbolic part of our equations. For this part we recover easily the incompressibility constraint in quasi-congested regions at the limit $\varepsilon \rightarrow 0$. Indeed, in regions where both $\gamma^{n}$, $\gamma^{n+1}<0$ we have on the approximate system $\rho_{\varepsilon}^{n}, \rho_{\varepsilon}^{n+1}>1-\varepsilon^{1 / \beta}$. Then the numerical scheme ensures that

$$
\left\|\partial_{x}\left(\rho_{\varepsilon} v_{\varepsilon}\right)^{n+1 / 2}\right\|_{L^{\infty}}=\left\|\frac{\rho_{\varepsilon}^{n+1 / 2}-\rho_{\varepsilon}^{n}}{\Delta t / 2}\right\|_{L^{\infty}} \leq \frac{2}{\Delta t} \varepsilon^{1 / \beta}
$$

and thus the incompressibility at the limit

$$
\partial_{x} v_{\varepsilon}^{n+1 / 2} \underset{\varepsilon \rightarrow 0}{\longrightarrow} 0
$$

Unfortunately we are not able to deduce the same for $u^{n+1}$ computed from

$$
\rho_{\varepsilon}^{n+1} \frac{u_{\varepsilon}^{n+1}-v_{\varepsilon}^{n+1}}{\Delta t / 2}-\partial_{x}\left(\nu\left(\rho_{\varepsilon}^{n+1}\right) \partial_{x} u_{\varepsilon}^{n+1}\right)=0
$$

Indeed the estimate of the $L^{2}$ norm of $\partial_{x} u_{\varepsilon}^{n+1}$, obtained by derivation with respect to $x$ and multiplication by $\partial_{x} u_{\varepsilon}^{n+1}$, involves in particular the integral

$$
\begin{aligned}
& -\int_{0}^{1} \partial_{x x}^{2}\left(\lambda_{\varepsilon}\left(\rho_{\varepsilon}^{n+1}\right) \partial_{x} u_{\varepsilon}^{n+1}\right) \partial_{x} u_{\varepsilon}^{n+1} \\
& \quad=\int_{0}^{1} \partial_{x} \lambda_{\varepsilon}\left(\rho_{\varepsilon}^{n+1}\right) \partial_{x} u_{\varepsilon}^{n+1} \partial_{x x}^{2} u_{\varepsilon}^{n+1}+\int_{0}^{1} \lambda_{\varepsilon}\left(\rho_{\varepsilon}^{n+1}\right)\left(\partial_{x x}^{2} u_{\varepsilon}^{n+1}\right)^{2}
\end{aligned}
$$

where the first integral cannot be bounded uniformly with respect to $\varepsilon$.

Nevertheless, the following numerical simulations seem to show that the incompressibility constraint is satisfied at the limit $\partial_{x} u_{\varepsilon}^{n+1} \rightarrow 0$ as $\varepsilon \rightarrow 0$ in the congested parts.

The designing of an Asymptotic Preserving scheme for the singular viscous system (8) will be the purpose of future work, it asks of course for a specific time-discretization of the singular viscous term. We also intend to propose a numerical discretization which does not involve the pressure $-\partial_{x} \gamma_{\varepsilon}$ in order to drop this term from the momentum equation which is a priori possible from theory as mentioned in the previous section.

\section{Numerical results}

For our numerical simulations, we do not have reference solution to compare with. Nevertheless, if initially we impose

$$
\rho^{0}(x)=0.7, \quad u^{0}(x)=\sin (2 \pi x), \quad \forall x \in[0,1]
$$

as represented on Figure 2, we can expect a concentration the fluid at the middle of the domain and the apparition of congestion if the viscosity is not too high. We have represented on Figures 3-4 the numerical solution computed from the solving of (22)-(23)-(24)-(25)-(26) for the following set of parameters

$$
\varepsilon=10^{-4}, \quad \beta=2, \quad \mu_{0}=0.5, \quad c_{\lambda}=0.5, \quad c_{\gamma}=1
$$

with

$$
\Delta x=5.10^{-3}, \quad \Delta t=10^{-4}
$$



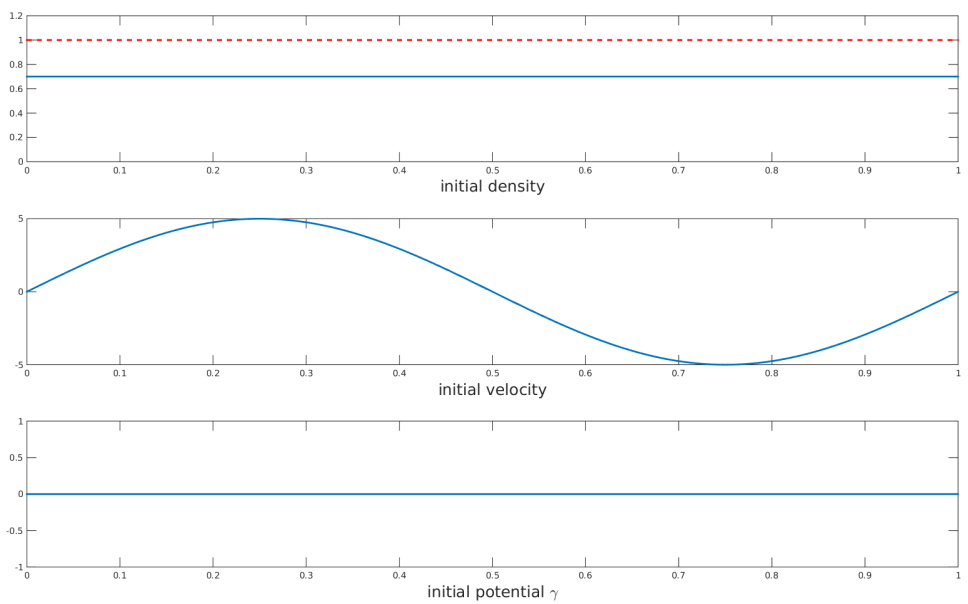

Figure 2. Periodic initial data

We observe on Figures 3-4 that the density effectively concentrates at the middle of the domain and flattens as it approaches the maximal constraint. A congested region (in red on the horizontal axis) appears where $\rho_{\varepsilon}>1-\varepsilon^{1 / \beta}$.

At the same time, the velocity becomes flat, almost 0 , in the congested part which show numerically that the incompressibility constraint is satisfied even with our splitting of the singular viscosity.

Finally we have represented the potential $\gamma_{\varepsilon}$ and $p_{\varepsilon}=-\lambda_{\varepsilon}\left(\rho_{\varepsilon}\right) \partial_{x} u_{\varepsilon}$. As expected from the theory, when $\varepsilon$ is small $\gamma_{\varepsilon}$ is activated only in the congested region as well as $p_{\varepsilon}$. Moreover, if at the beginning $\lambda_{\varepsilon}\left(\rho_{\varepsilon}\right)$ may take very large values, it is compensated after some time by the divergence of the velocity $\partial_{x} u_{\varepsilon}$.

\section{REFERENCES}

[1] B. Andreotti, Y. Forterre, O. Pouliquen. Granular media: between fluid and solid. Cambridge University Press, (2013).

[2] F. Berthelin. Existence and weak stability for a pressureless model with unilateral constraint. Mathematical Models and Methods in Applied Sciences, Vol. 12, 2, pp. 249-272, (2002).

[3] F. Bouchut, Y. Brenier, J. Cortes, J-F. Ripoll. Journal of NonLinear Science, Vol. 10, 6, pp. 639-660, (2000).

[4] L. Boudin. A solution with bounded expansion rate to the model of viscous pressureless gases. SIAM Journal on Mathematical Analysis 32.1, pp. 172-193, (2000).

[5] F. BOyER, P. FABRIE. Eléments d'analyse pour l'étude de quelques modèles d'écoulements de fluides visqueux incompressibles (Vol. 52). Springer Science \& Business Media (2005).

[6] A. Bertozzi, G. GüN, T. Witelski. Dewetting films: bifurcations and concentrations. Nonlinearity 14.6: 1569, (2001).

[7] D. Bresch, B. Desjardins. Some diffusive capillary models of Korteweg type. Comptes Rendus Mécanique, Vol. 332, 11, pp. 881-886, (2004).

[8] A. J. Chorin. A numerical method for solving incompressible viscous flow problems. Journal of computational physics 2.1, pp. 12-26, (1967).

[9] F. Cordier, P. Degond, A. Kumbaro. An Asymptotic-Preserving all-speed scheme for the Euler and Navier-Stokes equations. Journal of Computational Physics 231.17 pp. 5685-5704, (2012).

[10] P. Degond, F. Deluzet, A. Sangam, M. H. Vignal. An asymptotic preserving scheme for the Euler equations in a strong magnetic field. Journal of Computational Physics, 228(10), pp. 3540-3558, (2009).

[11] P. Degond, J. Hua, L. Navoret. Numerical simulations of the Euler system with congestion constraint. Journal of Computational Physics, Vol. 230, pp. 8057-8088, (2011).

[12] P. Degond, S. Jin, J.-G. Liu. Mach-number uniform asymptotic-preserving gauge schemes for compressible flows. Bull. Inst. More gMath. Acad. Sin. Vol. 2, pp 851-892, (2007). 

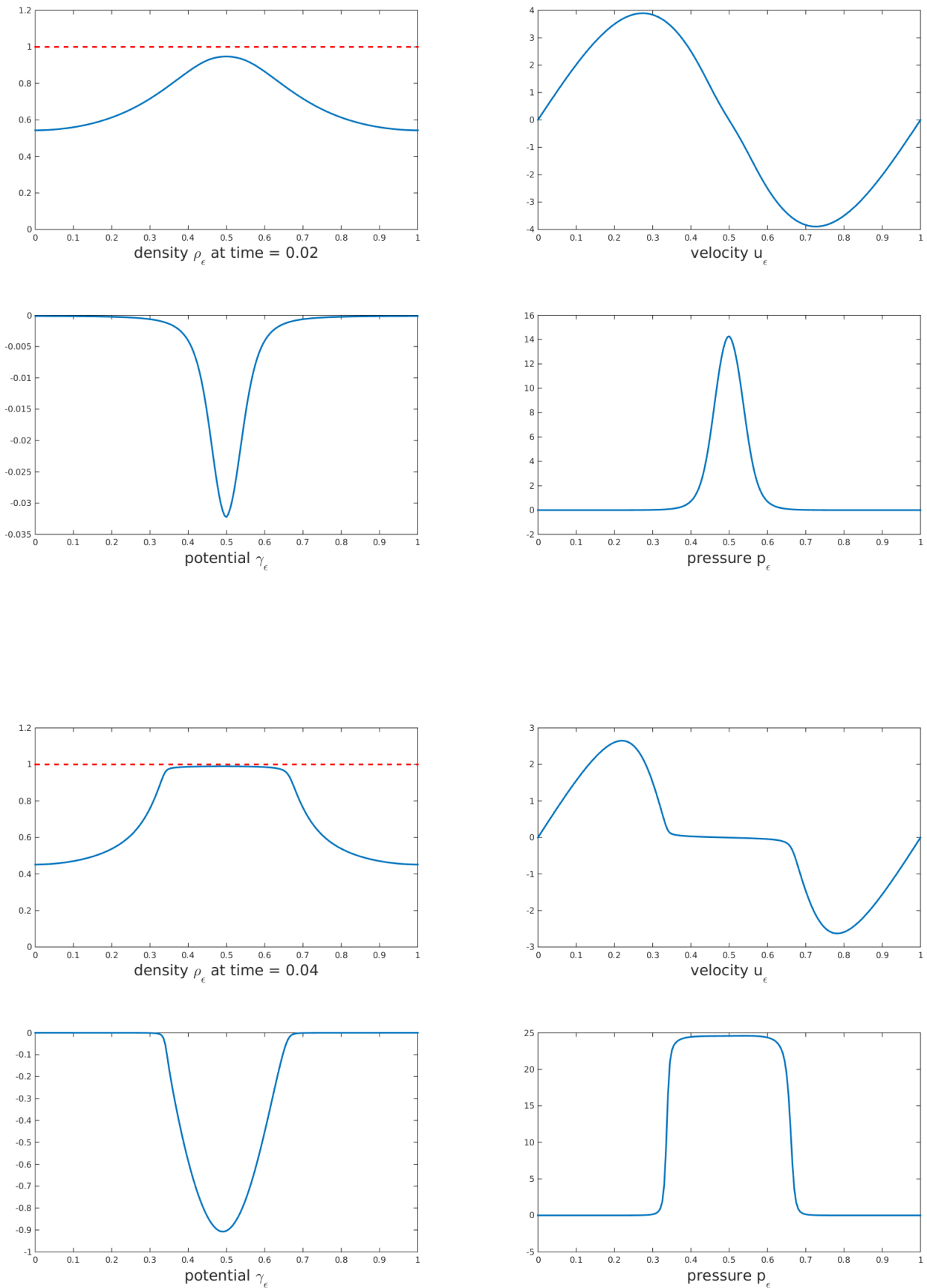

Figure 3. Variables $\rho_{\varepsilon}, u_{\varepsilon}, \gamma_{\varepsilon}$ and $p_{\varepsilon}=-\lambda_{\varepsilon} \partial_{x} u_{\varepsilon}$ at times $t=0.02$ and $t=0.04$ for $\varepsilon=10^{-4}, \beta=2, \mu_{0}=0.5, c_{\lambda}=0.5, c_{\gamma}=1$. 

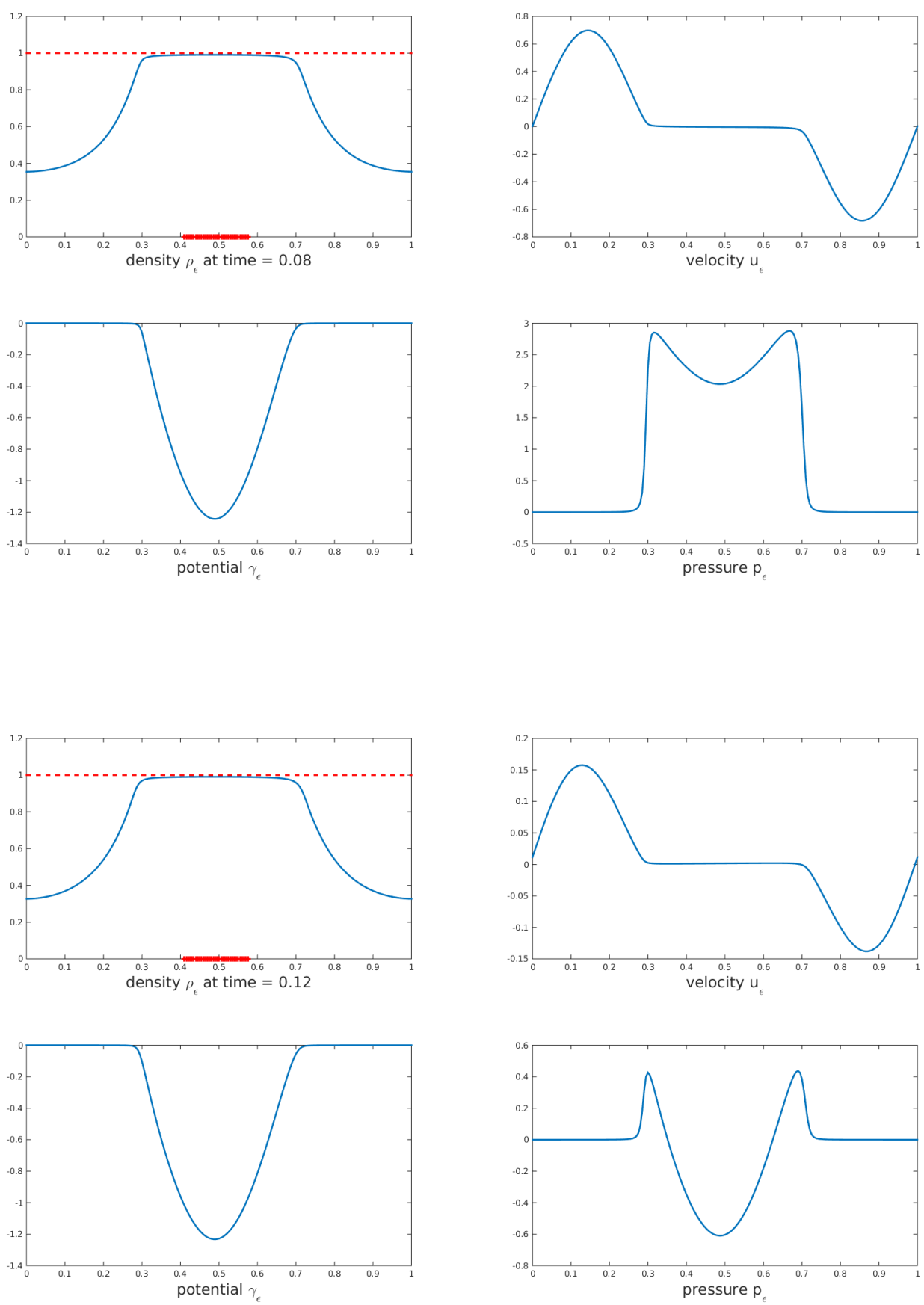

FiguRE 4. Variables $\rho_{\varepsilon}, u_{\varepsilon}, \gamma_{\varepsilon}$ and $p_{\varepsilon}=-\lambda_{\varepsilon} \partial_{x} u_{\varepsilon}$ at times $t=0.08$ and $t=0.12$ for $\varepsilon=10^{-4}, \beta=2, \mu_{0}=0.5, c_{\lambda}=0.5, c_{\gamma}=1$. The congested domain appears in red on the horizontal axis. 
[13] P. Degond, M. Tang. All speed scheme for the low mach number limit of the Isentropic Euler equation. ihal-00409851; (2009).

[14] F. Harlow, A. Amsden. A numerical fluid dynamics calculation method for all flow speeds. Journal of Computational Physics 8.2, pp. 197-213, (1971).

[15] B. Haspot. Porous media, Fast diffusion equations and the existence of global weak solution for the quasi-solution of compressible Navier-Stokes equations. arXiv preprint arXiv:1304.4502 (2013).

[16] R. Herbin, W. Kheriji, J.-C. Latche. Staggered schemes for all speed flows. ESAIM: Proceedings. Vol. 35. EDP Sciences, (2012).

[17] S. Jin. Efficient asymptotic-preserving (AP) schemes for some multiscale kinetic equations. SIAM Journal on Scientific Computing 21.2, pp. 441-454, (1999).

[18] R. KLein. Semi-implicit extension of a Godunov-type scheme based on low Mach number asymptotics I: One-dimensional flow. Journal of Computational Physics 121.2 pp. 213-237, (1995).

[19] A. Lefebvre-Lepot, B. Maury. Micro-macro modelling of an array of spheres interacting through lubrication forces Advances in Mathematical Sciences and Applications, Vol. 21,2, (2011).

[20] B. Maury A gluey particle model. Paris Sud working group on Modelling and Scientific Computing 2006-2007, ESAIM Proc., EDP Sci., Vol. 18, pp. 133-142, (2007).

[21] B. Maury, A. Preux. Pressureless Euler equations with maximal density constraint: a time-splitting scheme, hal-01224008, (2015).

[22] A. Mellet, A. Vasseur. On the barotropic compressible Navier-Stokes equations. Comm. Partial Diff. Eqns. 32(1-3), pp. 431-452, (2007).

[23] S. Noelle, G. Bispen, K. Arun, M. Lukacova-Medvidova, C.D. Munz. An asymptotic preserving all Mach number scheme for the Euler equations of gas dynamics. SIAM J. Sci. Comput (2014).

[24] C. Perrin. Pressure Dependent Viscosity Model for Granular Media obtained from Compressible Navier-Stokes Equations. To appear in Applied Mathematical Research Express.

[25] B. Perthame, F. Quirós, J. L. VÁzquez. The Hele-Shaw asymptotics for mechanical models of tumor growth. Archive for Rational Mechanics and Analysis 212.1, pp. 93-127, (2014).

[26] D. G. Schatffer. Instability in the evolution equations describing incompressible granular flow. Journal of differential equations 66.1, pp. 19-50, (1987).

[27] R. Temam. Sur l'approximation de la solution des équations de Navier-Stokes par la méthode des pas fractionnaires (II). Archive for Rational Mechanics and Analysis 33.5, pp. 377-385, (1969).

[28] E. TURKEL. Preconditioned methods for solving the incompressible and low speed compressible equations. Journal of computational physics 72.2 , pp. 277-298, (1987). 